\title{
Multi objective approach for leakage reduction in water distribution systems
}

\author{
R. Magini, I. Pallavicini \& D. Verde \\ Department of Hydraulics, Transportation and Roads, \\ University of Rome "La Sapienza", Italy
}

\begin{abstract}
This paper faces the problem of reducing leakages in water distribution systems (WDS) through dynamic heads control with pressure reduction valves (PRV). To achieve this aim a multi-objective optimization approach is followed for the location of control valves and for their setting during different working conditions. In particular, the objectives to be achieved are: 1) to minimize the leakages over all of the network, 2) to minimize the investment costs for the control devices. The objectives must be satisfied considering the variability of the nodal water demand. For a more realistic simulation of the WDS performance the implementation of a fully pressure-dependent leakage specification is required. The multi-objective optimization problem is tackled using a MOGA (Multi-Objective Genetic Algorithm) with a Pareto based approach. The hydraulic modelling of the network is performed with the software EPANET2. The nodal demands are considered to be uncertain input parameters and the most robust solution is also found. The procedure is here applied to the WDS of a small town not far from Rome afflicted by heavy losses. A reduction of about $50 \%$ of initial water losses is achieved.

Keywords: water distribution systems, leakage reduction, pressure control, multi objective optimization, genetic algorithms.
\end{abstract}

\section{Introduction}

Nowadays the problem of water losses control in WDS is more and more pressing owing to the growth of population, the increasing of water consumptions and at the same time because of the deterioration and the reduction of available drinkable water resources as a consequence of pollution and climate changes. Leakages in urban water networks can be a very high percentage of the 
supplied water. In some cases in Italy they can be even more than 50\%. This lost volume represents an economic damage not only for the cost of water pumping and treatment but even because it makes necessary investments into systems capacity expansion or in searching for other water sources. Losses are usually divided into 'apparent losses', including unauthorised consumptions by illegal connections and metering inaccuracies, and 'real or physical losses' produced by all types of leaks, burst or overflows, which are due to the state of degradation of the networks and to their bad management. Usually all the methods of leak detection are expensive, complex and time consuming. Physical losses, moreover, sometimes manifest themselves with local huge leakages, sometimes they are the sum of small leakages distributed all over the network. Then, it is necessary to define a critical threshold of the leakage over which the benefits from the detection and repairing are greater than the costs. The level of this threshold depends on the economic value of the resource, including all the costs for drawing, conveying, treating, pumping, etc. The threshold increases as the number of leakage points increases, that is, when it is related to 'distributed leaks'. In this case leak detection and repairing operations are often non realistic and too expensive also if the volume of the water losses is relevant. Then it may be better to plan medium-long term rehabilitation policies of the network combined with suitable management policies for controlling pressure heads in the system. Pressure regulation can be achieved by means of pressure reduction valves (PRV) remotely controlled, whose purpose is to decrease excessive pressures, ensuring the service conditions (Reis et al [1], Lumbers and Vairavamoorthy [2], Araujo et al [3]).

In this paper a method to determine the optimal number and location of the valves and their optimal setting for different demand scenarios is presented and the case-study of a little town not far from Rome has been considered. At this purpose the network simulation has been carried out considering the leakages as pressure-driven through the 'emitter function' of EPANET2 (Araujo et al [4], Burrows and Zhang [5], Tabesh et al [6]).

Firstly a calibration procedure for assessing nodal demands and distributing properly leakages throughout the network has been performed. The successive optimization analysis for the location of control valves and for their setting during different working conditions has been tackled using a MOGA (MultiObjective Genetic Algorithm) with a Pareto based approach. Finally a robust optimization analysis, aimed at finding the most robust solution in respect to non deterministic parameters, has been also performed. In particular uncertainty of water demands has been considered.

\section{Calibration and assessment of leakage and demand}

In order to treat water losses as pressure dependent quantities, the calibration of the WDS simulation model has been performed concentrating leaks at the nodes and considering their flow $Q^{\text {leak }}$ as a function only of the local pressure $p$ according to the expression:

$$
Q^{\text {leak }}=K p^{n}
$$


with $n=1.18$, as experimental experiences have shown (Goodwin [7]). The presence of the leakage term modifies the continuity equation of the generic node $i$ as follows:

$$
\sum_{p=1}^{N_{i}} Q_{p}-D_{i}-K_{i} p_{i}^{n}=0
$$

where $Q$ indicates the flow in the $p^{\text {th }}$ trunk connected to the node $i$ and $D$ the water demand at the node. For the parameter $K$, called emitter coefficient, the following expression has been considered:

$$
K_{i}=c \sum_{p=1}^{N_{i}} \frac{1}{2} L_{p}
$$

where $L$ is the length of the $p^{\text {th }}$ trunk connected to the node $i$ and $c$ an unknown coefficient valid for the entire network.

To calibrate the parameter $c$, a procedure presented by Araujo [4] has been followed. This method requests to know flow and pressure measurements in the inlet and outlet nodes of the network. The difference between the inlet and the outlet flow represents the discharge $Q^{\text {net }}$ flowing into the network. For the evaluation of the coefficient $c$ an estimation of the percentage $\% F$ of the global leakage discharge at the minimum night flow (MNF) time has been also carried out. Then the equality between the supposed total leakage flow at the MNF time and the sum of leakage discharges modelled with eqn (1) has been imposed:

$$
\left[Q_{\text {tot }}^{\text {leak }} \equiv \% F Q^{\text {net }}=\sum_{i}^{N} K_{i}(c) p_{i}^{1.18}\right]_{M N F} .
$$

The estimation of the coefficient $c$ can be carried out with an iterative procedure. In this work an optimization problem solved with GA coupled with the hydraulic model of the network has been adopted.

The coefficient $c$ has been estimated for the MNF hour but it has been supposed constant at any consumption conditions. For this reason it is necessary to calculate a coefficient $f_{t}$ for each time interval $t$ in which the day is split (i.e. in 24 hours) in order to satisfy the following equation:

$$
Q^{n e t}(t)=f_{t} \sum_{i=1}^{N} D_{i}(t)+\sum_{i=1}^{N} K_{i} p_{i}^{1.18}(t) .
$$

This strategy enables a better assessment of the nodal demands, taking into account the measurements available in the inlet and outlet nodes and considering the presence of leakages too. Also in this case the estimation of the coefficients $f_{t}$ can be carried out with a GA coupled with the hydraulic model of the network.

Once the $c$ and $f_{t}$ coefficients have been estimated the WDS is completely characterized. 


\section{Optimization procedure}

An optimization procedure has been set up for finding the optimal number of PRVs, their best location inside the network and their setting for different demand scenarios with the aim of minimizing the leakages and the costs (number of valves). The problem constraints were defined by the lowest acceptable nodal pressures at each node in order to satisfy the service conditions.

The optimization process has been divided into two phases. In the first one, denoted 'designing phase', the optimal position and number of valves has been found. In this phase the MNF condition, for which the maximum value of leakages is expected, has been considered.

The opening degrees of each valve define the number of decision variables. Their number is equal to the number of pipes of the network and each of them can assume 11 integer values in the range $0-10$. The value 0 corresponds to the absence of the valve, while the other values define different opening settings. A set of $L$ binary counters $x$ identifies the number of installed valves. Then the two objective functions are:

$$
\begin{gathered}
\text { min_Leakage }=\sum_{i}^{N} K_{i} P_{i}^{1.18}, \\
\text { min_Valve_number }=\sum_{k=1}^{L} x_{k} .
\end{gathered}
$$

The problem constraints are represented by the hydraulic equations of the network and by the minimum pressure levels to be assured at each node. To solve this problem, an evolutionary MOGA algorithm, using specific operators such as a multi directional crossover and elitism, has been employed and combined with the hydraulic solver Epanet2.

In this phase also the valve opening degrees have been implicitly optimized. The goal of this phase was to find the best configuration that gives the largest reduction of leakages throughout the whole day. However the optimization has been performed considering only the MNF hour, when the greatest value of water losses should be expected.

A multi-objective optimization problem yields a set of non-dominated solutions, which form the Pareto front in the objective functions space. It is then necessary to select the best one with suitable criteria.

The second phase, denoted 'management phase', has been carried out in order to determine the best setting of the installed PRVs for the different demand scenarios of a typical day. Now the only objective to be considered is the minimization of the leakages, eqn (6a). Moreover the number of decision variables is obviously reduced to the number of PRVs previously determined. Different optimizations have been performed for the 24 hours. 


\section{Robust design}

Robust design is a procedure of optimization, which takes into account that some parameters of the problem can be affected by uncertainty. In particular here we have considered water demands at each node as stochastic variables.

In an optimization problem a robust solution must be not only optimal, but also not too sensible to variations of the uncertain parameters. The method used was based on a Montecarlo generation of different scenarios for any possible solution evaluated by the GA. A mean value of the fitness function and of the other outputs are then considered. The solution is robust if the mean value of the fitness function is optimized and its standard deviation minimized.

This methodology has been applied to the second phase optimization problem. Water demands at each node have been considered to be normal distributed, with a mean value deriving from a study on the characteristics of the end users (Pallavicini et al [8]). This value is the same used both in the first and in the second phase. The standard deviation has been considered variable in the different hours of the day, following experimental studies on instantaneous water demand (Guercio et al [9]).

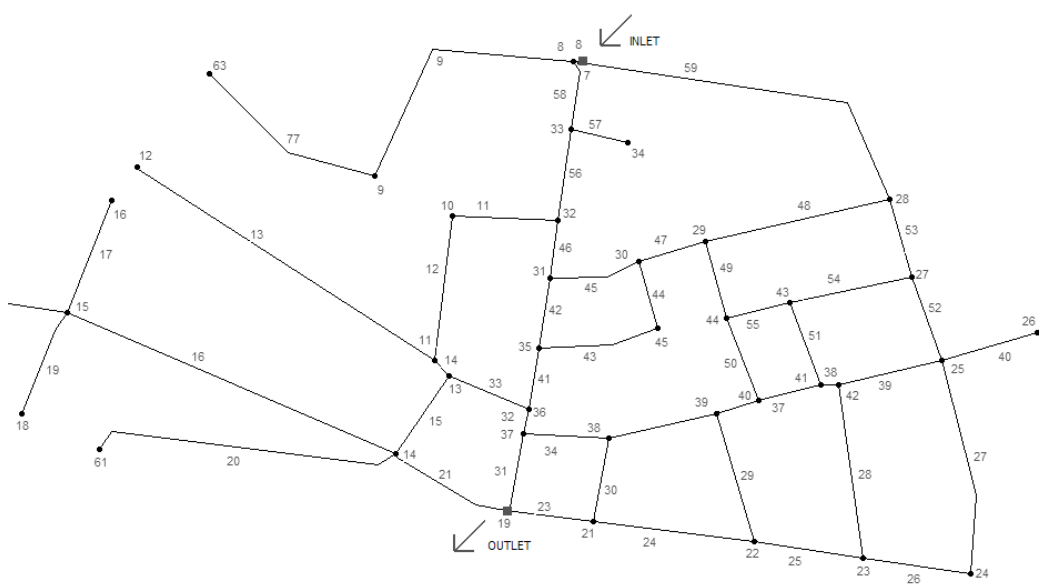

Figure 1: $\quad$ The case study WDS.

\section{Case study}

The application of the discussed procedure has been carried out on the real water distribution network of Piedimonte S. Germano, a small town of about 4700 inhabitants in the south of Latium. This network is affected by serious water losses. Actually only the portion of the network serving 714 equivalent users and supplying the oldest part of the town has been considered, fig. 1. The network, mainly composed by cast iron pipes and partly by HDPE pipes, is situated at a mean elevation of $112 \mathrm{~m}$ asl and is served by a unique reservoir 
located at $180 \mathrm{~m}$ asl. The inlet and outlet nodes are monitored with electromagnetic flow meters and pressure transducers connected to data loggers.

\subsection{Calibration results}

The results of this preliminary study are summarized in fig. 2. In particular in fig. 2(a) the values of the difference between the inlet and the outlet measured flows, $Q^{\text {net }}$, and of the measured pressure at inlet node are represented for 24 hours. In the same figure is also reported the daily global demand pattern $D$, obtained by means of an accurate description of the different kind of users at each network node. From the $Q^{\text {net }}$ curve it is evident that the MNF occurs at 3:00 am. At this time the percentage $\% F$ of the leakages has been fixed equal to $95 \%$ of $Q^{\text {net }}$. In this figure the leakages curve has been obtained from the difference between the $Q^{\text {net }}$ and the demand without considering any dependence on the pressure.

The calibration procedure has yielded the coefficient $c$ and the $N$ emitter coefficients $K$ calculated according to eqn (3). Then the global demand has been corrected using the coefficients $\mathrm{ft}$. The final results of the calibration are summarized in fig. 2(b) where the modified behaviour of the demand and leakages can be observed. In this figure leakages follow a more realistic behaviour as they are related to pressure according to eqn (1). The same calibration procedure has enabled to estimate the distribution of leaks at each node and at different hours. The main result of this calibration process has been the estimation of the daily water volume lost that is equal to $196 \mathrm{~m}^{3}$. Considering equal to $357 \mathrm{~m}^{3}$ the water volume entering the network, leakages reach the very large percentage of about $55 \%$.
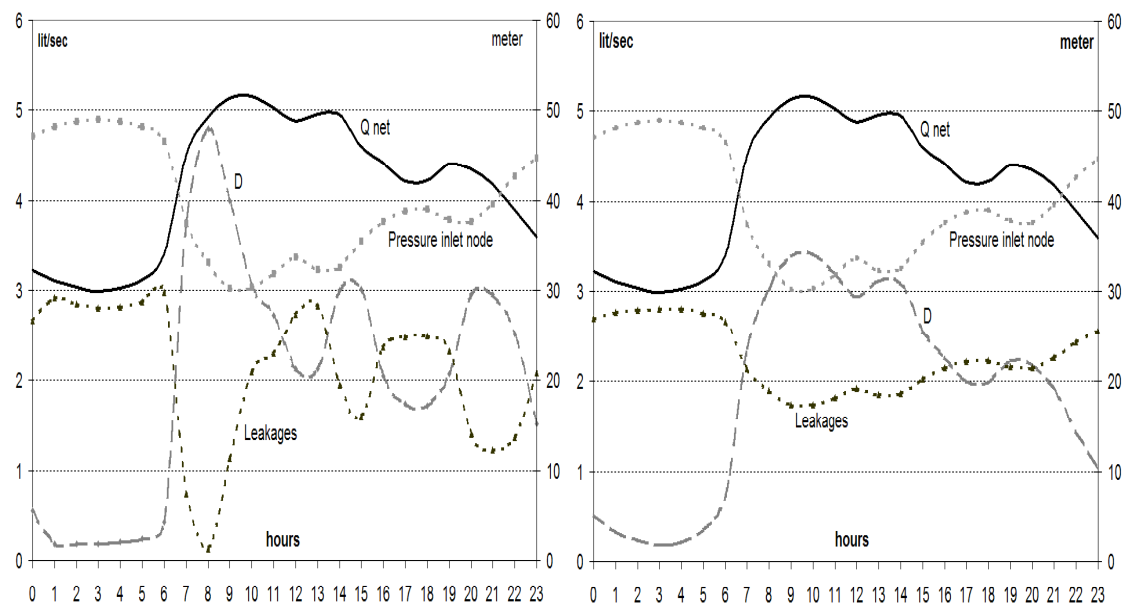

01234567891011121314151617181920212223

Figure 2: (a) (left) available data; (b) (right) calibration's results. 


\subsection{First phase results}

The multi-objective problem, aimed at determining the number of the PRVs and their best position, has been solved by means of the MOGA II algorithm implemented by the software mode Frontier [10]. Some $\mathrm{C}++$ utilities have been developed to make MOGA II interact with the hydraulic solver Epanet2.

It is interesting to mention that, because the number of decision variables are 51 (number of pipes) and each one can assume 11 different values (opening degrees), the search space dimension is equal to the huge value of $11^{51}$. The MOGA has been executed with a population of 1000 individuals and with 50 generations, for a total of 50000 solutions evaluated. In fig. 3 the space of the objective functions with some of the obtained solutions is represented. Focusing on the Pareto frontier it is easy to deduce that the introduction of more than 4 valves is useless because the consequent marginal reduction of the losses would be negligible. The location of the 4 valves of the best solution (circled point), is represented in the fig. 4. Their position is close to the inlet node because the network is characterized by high pressures owing to the reservoir elevation.

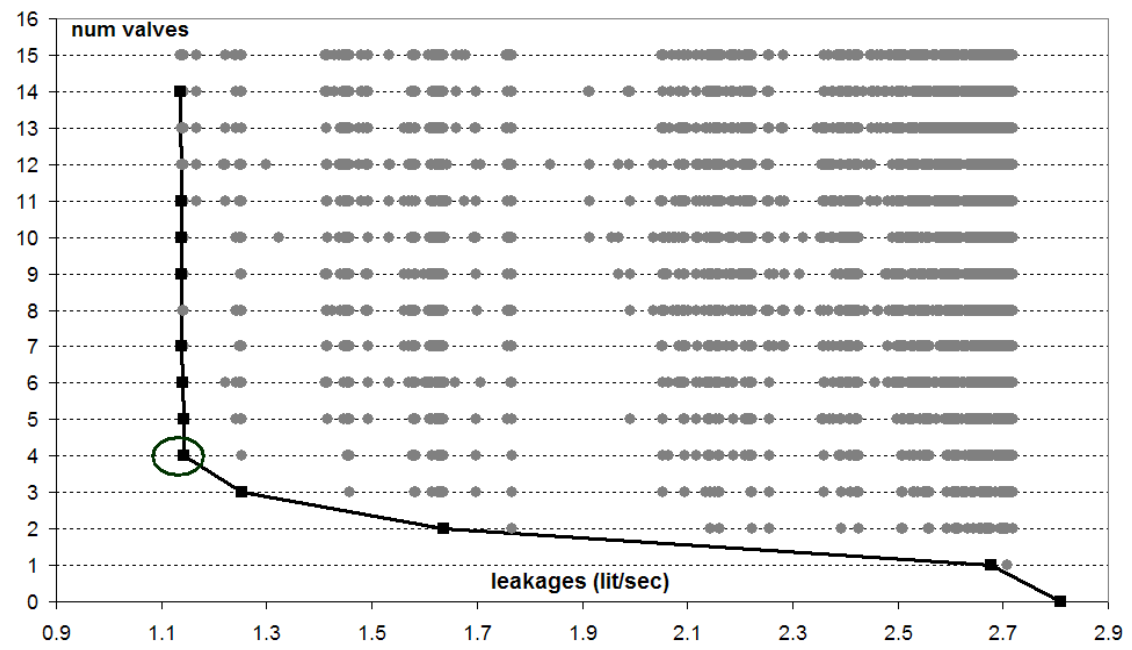

Figure 3: Results on objective functions space, Pareto Front.

\subsection{Second phase results}

The second phase has been concerned with the optimization of the opening degrees of the four installed valves during the 24 hours with the only objective to minimize water losses. This problem is single-objective but it has been solved using the MOGA II algorithm anyway. In this case the solutions space has the dimension of $11^{4}$. The 24 optimizations processes have been performed with a 
population of 150 individuals and 10 generations, therefore the algorithm has found the solution by evaluating 1500 possible solutions. The effect of the four installed valves and their dynamical setting is summarized in fig. 5. The comparison with fig. 2(b) shows that leakages are drastically reduced and that they are almost constant during the whole day. The effect of the valves in fact maintains the pressures close to the minimum requested value. The final result is that the average supplied volume of water in a day is reduced to about $256 \mathrm{~m}^{3}$. This means that a reduction of about $50 \%$ of the lost volume has been achieved. Leakages are reduced to $95 \mathrm{~m}^{3} /$ day corresponding to about $37 \%$ of the total entering volume.

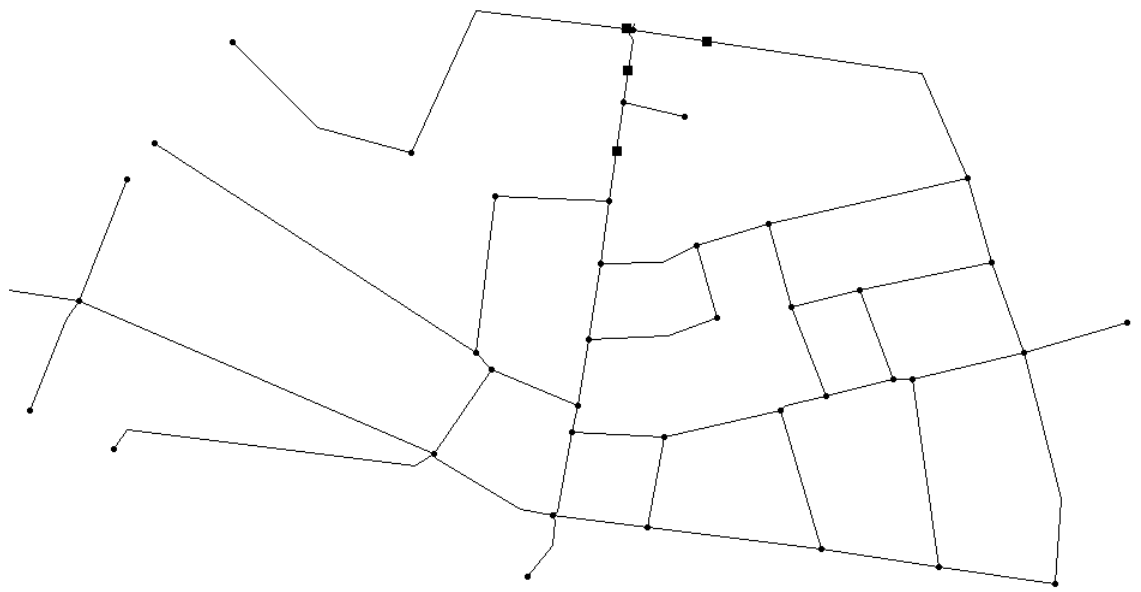

Figure 4: Position of the four valves inside the network.

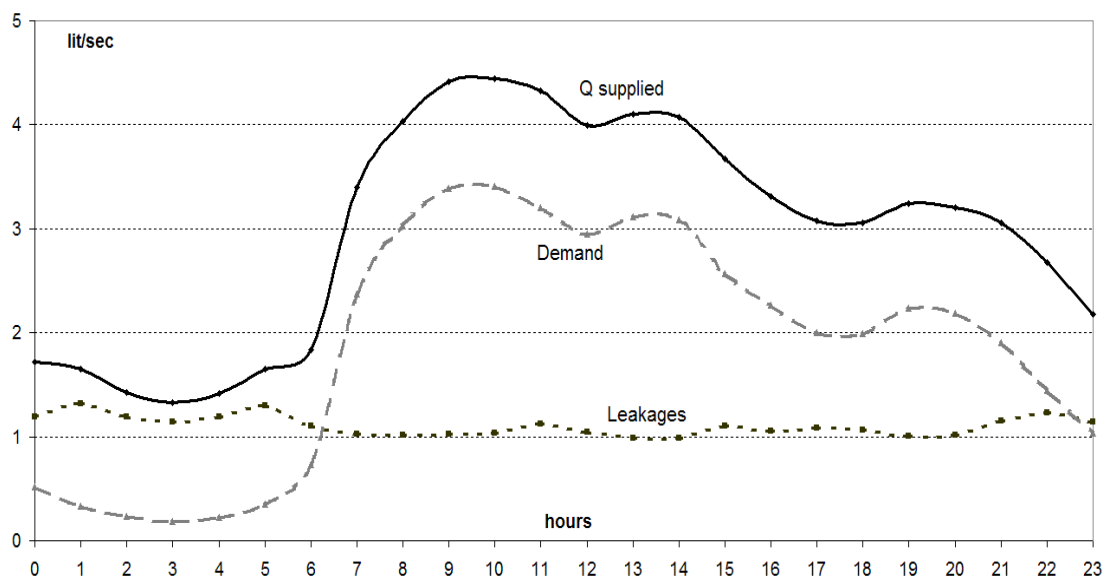

Figure 5: WDS daily average performance with the four valves. 


\subsection{Robust design analysis}

The valve setting optimization has been performed at the two extreme demand scenarios of 3:00 a.m. and 9:00 a.m., considering for each node a normal distribution of the demand with a standard deviation respectively equal to $10 \%$ and $5 \%$. These values are about twice the results obtained from experimental data (Guercio et al [9]). With the Montecarlo technique 15 different demand scenarios have been generated. The standard deviation of the objective function (min_leakages) has been fixed equal to $2 \%$ of the result obtained in the optimization of previous phases.

In both cases the valve setting obtained considering the robust design coincides with the one previously obtained with a deterministic approach.

\section{Conclusions}

In this paper a multi-objective approach for the optimal location and the optimal setting of pressure control valves in a water distribution network has been presented. The multi-objective optimization problem has been tackled using MOGA with a Pareto based approach. The methodology has been applied to a district of the water distribution network of a small town in the south of Latium (Italy) characterized by water losses exceeding the $50 \%$ of the entering water volume. A calibration process has been firstly carried out in order to obtain a complete and realistic characterization of water demand and leakages. In particular leakages have been considered strongly related to pressures. The successive optimization procedure has been split into two phases. In the first one the optimal location and the best number of valves has been determined at MNF condition, when leakages are expected to be largest. The multiple objective has been here represented by the minimization of the total leakage volume all over the network and the minimization of the costs. The optimal solution suggests the installation of four valves. In the second phase the optimal setting of the installed valves has been defined for the whole day. A reduction of about $50 \%$ of the lost volume has been achieved and leakages decrease to about $37 \%$ of the total entering volume. Finally a robust design analysis was also carried out in order to examine the sensitivity of the optimal solutions with respect to uncertainty of water demand.The good results achieved could be further improved introducing a forecasting model for water demand to be integrated in a real time system for the optimal management of the valves. At this aim it should be advisable to design an optimal monitoring system for flow and pressure which could enable during the 'calibration phase' a better characterization of the leakage and demand distribution, and in the 'management phase' could support the real time control.

\section{References}

[1] Reis F.R., Porto R.M. \& Chaundhry F.H., Optimal location of control valves in pipe networks by genetic algorithm. J. Water Resour. Plng and Mgmt., ASCE 123 (6), 1997, pp. 317-326. 
[2] Lumbers J. \& Vairavamoorthy K., Leakage reduction in water distribution systems: optimal valve control. J. Hydraulic Engineering, ASCE 124 (11), 1998, pp. 1146-1153.

[3] Araujo L.S., Coelho S.T. \& Ramos H.M., Optimization of the use of valves in a network water distribution system for leakage minimization. Proc. of Int. Conf. on Advances in Water Supply Management, London, pp. 97-107, 2003.

[4] Araujo L.S., Coelho S.T. \& Ramos H.M., Estimation of distributed pressure-dependent leakage and consumer demand in water supply networks. Proc. of Int. Conf. on Advances in Water Supply Management, London, pp. 119-128, 2003.

[5] Burrows R. et al. \& Zhang J. et al., Introduction of a fully dynamic representation of leakage into network modelling studies using Epanet. Proc. of Int. Conf. on Advances in Water Supply Management, London, pp. 109-118, 2003.

[6] Tabesh M., Asadiani Yekta A.H. \& Burrows R., Evaluation of UFW and real losses in WDS by hydraulic analysis of the system considering pressure dependency of leakage. Proc. of CCWI, Exeter, pp. 125-130, 2005.

[7] Goodwin S.J., The result of the experimental program on leakage and leakage control. Technical Report TR 154, W.R.C., Swindon, UK, 1980.

[8] Pallavicini I, Magini R \& Guercio R., Assessing the spatial distribution of Pressare Heads in Municipal Water Networks, Proc. of CCWI, Exeter, pp. 257-262, 2005

[9] Guercio R., Magini R. \& Pallavicini I., Monitoraggio ed analisi di consumi idrici residenziali. Proc. of XXIX Convegno Idraulica $e$ Costruzioni idrauliche, Trento, Vol.C, pp.271-278, 2004. In Italian.

[10] modeFrontier, http://frontier.enginsoft.it/software/index.html 